\title{
A Variational Approach for Denoising Hyperspectral Images Corrupted by Poisson Distributed Noise
}

\author{
Ferdinand Deger ${ }^{1,2}$, Alamin Mansouri ${ }^{1}$, Marius Pedersen ${ }^{2}$, \\ Jon Yngve Hardeberg ${ }^{2}$, and Yvon Voisin ${ }^{1}$ \\ 1 Le2i - Université de Bourgogne, Auxerre, France \\ ferdinand.deger@u-bourgogne.fr \\ ${ }^{2}$ Norwegian Colour and Visual Computing Laboratory - Gjøvik University College, \\ Gjøvik, Norway
}

\begin{abstract}
Poisson distributed noise, such as photon noise is an important noise source in multi- and hyperspectral images. We propose a variational based denoising approach, that accounts the vectorial structure of a spectral image cube, as well as the poisson distributed noise. For this aim, we extend an approach for monochromatic images, by a regularisation term, that is spectrally and spatially adaptive and preserves edges. In order to take the high computational complexity into account, we derive a Split Bregman optimisation for the proposed model. The results show the advantages of the proposed approach compared to a marginal approach on synthetic and real data.
\end{abstract}

\section{Introduction}

Multi- and Hyperspectral imaging (HSI) combine digital imaging and spectroscopy, and has numerous applications in remote sensing, mineralogy, cultural heritage documentation etc. The technology acquires radiometric information for every pixel in an image. The discrete number of spectral bands form, in combination with the spatial information a 3-dimensional data cube.

Spectral images contain noise, which impacts the precision of further processing steps, such as unmixing, classification, reflectance estimation [10] or compression 4]. The image noise includes signal-dependent components, such as photon noise, and signal-independent components such as dark noise or fixed pattern noise. Previous research [1,7,12] identified the photon noise, as the most relevant noise contribution in HSI. Hyperspectral scanners are sophisticated, individually calibrated devices with a high signal to noise ratio (SNR). Signal-dependent noise components are proportional to the signal amplitude, and are therefore more important in images with a high SNR [12. HSI applications, outside the field of remote sensing allow to repeat a single measurement multiple times, and increase the SNR further [1]. Calibrated scanners allow a compensation for the relative responsivity of detector elements and therefore suppress fixed noise patterns [9]. Common applications have a low number of photons, due to a weak signal or a large distance, which leads to a photon-limited regime [7]. 
To reduce noise in a photon-limited hyperspectral image, different approaches have been proposed. Othman and Qian [12] published a hybrid spatial-spectral derivative-domain wavelet shrinkage, that benefits from the dissimilarity of the signal regularity in the spatial and the spectral dimensions of hyperspectral images. Krishnamurthy, Raginsky, and Willett [7] partitioned a hyperspectral cube into anisotropic cells and maximised a penalised log likelihood criterion.

Total Variation (TV) image denoising has recently been applied to HSI. Yuan, Zhang, and Shen [15] employed a spectral-spatial adaptive Rudin-Osher-Fatemi (ROF) [13] model and showed good performance, especially when the noise level is different in each band. Variational image denoising is a very effective and efficient denoising approach, that preserves edges and has been applied to many imagery applications. However, like most variational approaches, the spectralspatial adaptive approach in [15] is based on a signal-independent ROF model, assuming an additive gaussian noise model. For monochrome images, TV denoising has been extended to other noise distributions. Le, Chartrand, and Asaki [8] proposed a ROF model for poisson distributed noise in monochromatic images. Due to the negative log likelihood used in the formulation, such an extension is difficult to calculate and different approaches for an optimisation have been proposed [6, 8, 16.

In oder to account for both, the vectorial structure of the HSI cube, and the signal-dependent poisson distributed noise, we propose a variational approach for denoising HSI. To our knowledge it is the first extension of a poisson distributed ROF model to HSI.

The rest of this paper is organised as follows. In Section 2 we describe the ROF model for poisson distributed noise and introduce an optimised TV regularisation terms for HSI. As the proposed ROF model shows a high computational complexity, we employ a split Bregman optimisation. In Section 3 we evaluate the proposed method on a synthetic dataset quantitatively and qualitatively and show the advantages of an optimised TV regularisation terms for the HSI cube. The proposed approach is then applied to real data and we conclude in Section 4

\section{Proposed Method}

\subsection{Problem Formulation}

Assume we have an original hyperspectral image $u$ and a measurement $f$, distorted by poisson noise,

$$
f \sim \operatorname{Poisson}(u)
$$

where $u$ and $f$ are both of dimensions $M \times N \times B$, in which $M$ and $N$ are the spatial dimensions of the image, and $B$ represents the spectral band-number. As developed in [8], the estimation of a reconstructed image $\hat{u}$ can be described by a MAP estimate

$$
\hat{u}=\arg \max _{u} P(f \mid u) P(u) .
$$


The poisson distribution in a discrete image can be formalised as

$$
P(f \mid u)=\prod_{i}^{M N B} \frac{e^{-u_{i}} u_{i}^{f_{i}}}{\left(f_{i}\right) !}
$$

and the prior $P(u)$ depends on the TV seminorm

$$
P(u)=\exp \left(\lambda\|u\|_{\mathrm{TV}(\Omega)}\right),
$$

where $\lambda$ is the corresponding regularisation parameter and $\Omega=M \times N \times B$ is the image domain. Equation (2) is solved by minimising $-\log (P(f \mid u) P(u))$, which can be simplified to the following ROF problem

$$
\hat{u}=\arg \min _{u} \sum_{i}^{M N B}\left(u_{i}-f_{i} \log u_{i}\right)+\lambda\|u\|_{\mathrm{TV}(\Omega)} .
$$

The TV term in (4) was originally developed for greyscale images [13], as the sum of the image gradient magnitudes. For a HSI cube it can be written as a marginal approach TV (MTV)

$$
\|u\|_{\mathrm{MTV}}=\sum_{i}^{M N B} \sqrt{\left(\nabla_{x} u\right)_{i}^{2}+\left(\nabla_{y} u\right)_{i}^{2}}
$$

where $\nabla_{x}$ and $\nabla_{y}$ are the discrete horizontal and vertical derivation in the image plane $M \times N$.

\subsection{Spectral Total Variation Seminorm}

A vectorial ROF model was developed for colour images [3] and extended to HSI [15, by coupling the gradients of all channels at every image location. The vectorial TV seminorm (VTV) is denoted as

$$
\|u\|_{\mathrm{VTV}}=\sum_{i}^{M N} G_{i}, G_{i}=\sqrt{\sum_{j}^{B}\left(\nabla_{x} u\right)_{i, j}^{2}+\left(\nabla_{y} u\right)_{i, j}^{2}} .
$$

The norm is based on the gradients of all bands, which increases the smoothing factor for band gradients, that are higher than the average. By which highernoise bands are smoothed stronger. In [15] this model was extended by spatial weighting factor $W_{i}$, exploiting the normalised local strength of $G_{i}$ at the position $i$, which is defined as

$$
W_{i}=\frac{\left(1+\mu G_{i}\right)^{-1}}{\frac{1}{M N} \sum_{k}^{M N}\left(1+\mu G_{k}\right)^{-1}}
$$


where $\mu$ is a positive constant factor. For a higher gradient $G_{i}$, the corresponding weight $W_{i}$ becomes smaller in the range of $[0,1]$, and for smaller gradients viceversa. VTV is extended to a spatial-spectral adaptive TV seminorm (SSATV) by multiplying this weighting factor

$$
\|u\|_{\text {SSATV }}=\sum_{i}^{M N} W_{i} G_{i} .
$$

The different TV-seminorms MTV, VTV, and SSATV can be applied to the ROF model for poisson distributed noise (5). For SSATV the proposed ROF model becomes

$$
\hat{u}=\arg \min _{u} \sum_{i}^{M N} W_{i} G_{i}+\lambda \sum_{i}^{M N} \sum_{j}^{B}\left(u_{i, j}-f_{i, j} \log u_{i, j}\right) .
$$

In this model, the logarithmic data term accounts for the poisson distributed noise and the regularisation for HSI.

\subsection{Optimisation}

To solve the ROF problem (10), we use a Split-Bregman algorithm, developed by Goldstein and Osher 11, to efficiently calculate $L^{1}$ and more specifically TV regularisations. The main idea is to apply an operating splitting and transforming the original problem into a constrained minimisation problem, that can be solved with Bregman iterations [2]. This optimisation has been applied to TV denoising 11, and the HSI spatial-spectral adaptive denoising in the case of Gaussian noise [15. Due to the logarithmic data term, we apply an extension [6] with two auxiliary variables. In a first step the ROF problem (10) is rewritten as constrained minimisation, that is subject to both the auxiliary variables $d$ and $z$

$$
\begin{array}{r}
\arg \min _{d, z, u} \sum_{i}^{M N} W_{i} \sqrt{\sum_{j=1}^{B}\left(d_{i, j}\right)^{2}}+\lambda \sum_{i}^{M N} \sum_{j}^{B}\left(z_{i, j}-f_{i, j} \log z_{i, j}\right) \\
\text { subject to }: \quad d=\nabla u_{i, j}^{2}, \quad z=u .
\end{array}
$$

This can be transferred into an unconstrained minimisation problem, which is described in [6] for monochromatic images. An iterative algorithm solves for the auxiliary variables $d, z$, as well as the reconstruction of the noiseless image $u$. These three subproblems can be easily solved, which reduces the overall complexity from $O\left(N^{3}\right)$ to $O\left(N^{2}\right)$ [15. The $u$-subproblem is solved as a fast single iteration of the Gauss-Seidel algorithm, $d$-subproblem is solved by a shrinkage operator and $z$-subproblem decouples over $i, j$. We follow the algorithm described in [6] and account spectral images with a modified shrinkage operator in the $d$-subproblem similar to [15]. 


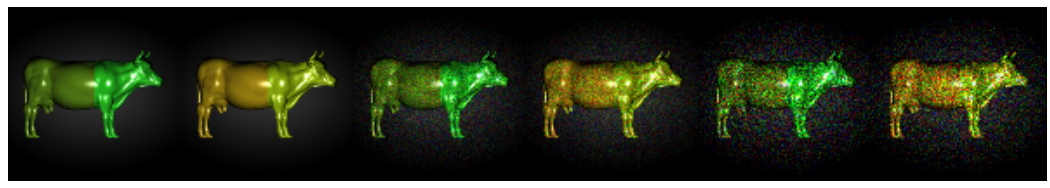

(a) original

(b) $\sigma_{3}=0.04$

(c) $\sigma_{5}=0.08$

Fig. 1. Two out of the 24 cows from the Metacow image [5], illuminated with a D65 illuminate. Different noise levels are shown. To visualise the HSI we assigned band 460 $\mathrm{nm}$ to the blue, Band $565 \mathrm{~nm}$ to the green and $615 \mathrm{~nm}$ to the red channel.

\section{Experimental Evaluation}

\subsection{Synthetic Dataset}

The computer generated Metacow [5] has its origins in the field of colour imaging, it shows 24 cows with different spectral surfaces, and is freely available. The image is a noiseless, high contrast HSI, containing spectral reflectance of 77 bands from $380 \mathrm{~nm}$ to $760 \mathrm{~nm}$. We multiplied the image with a spatially uniform D65 illumination to simulate radiance values and scaled the image to a range of 0 to 1 . To apply different levels of noise the image is scaled to a higher magnitude by a factor $a$, before adding the poisson distributed noise

$$
f_{i}=\operatorname{Poisson}\left(u_{i} / a\right) a, \quad a=\frac{\sigma^{2}}{\operatorname{mean}(u)}
$$

where $\sigma$ is the noise intensity in the range from $\sigma_{1}=0.005$, to $\sigma_{6}=0.1$. Different noise levels are visualised in Fig 1 .

We evaluated different TV terms, that can be used in the proposed ROF model. MTV treats the HSI as a stack of monochromatic images, while VTV and SSATV account the HSI cube. For the evaluation we use two metrics. The peak signal to noise ration (PSNR) between the groundtruth $u$ and an estimation $\hat{u}$

$$
\operatorname{PSNR}(u, \hat{u})=10 \log _{10}\left(\frac{\max (u)}{\operatorname{MSE}}\right), \quad \operatorname{MSE}=\frac{1}{M N B} \sum_{i}^{M N B}\left(u_{i}-\hat{u}_{i}\right)^{2},
$$

and the structural similarity (SSIM) [14] index

$$
\operatorname{SSIM}(u, \hat{u})=\frac{\left(2 \mu_{u} \mu_{\hat{u}}+c_{1}\right)\left(2 \sigma_{u, \hat{u}}+c_{2}\right)}{\left(\mu_{u}^{2}+\mu_{\hat{u}}^{2}+c_{1}\right)\left(\sigma_{u}^{2}+\sigma_{\hat{u}}^{2}+c_{2}\right)},
$$

where $\mu$ is the average and $\sigma^{2}$ is the variance, both in a local neighbourhood. The constants $c_{1}$ and $c_{2}$ stabilise the division with weak denominator and are set to fixed ratio of the maximum image value. Both metrics are applied band-wise and globally. PSNR is defined for both 2- and 3-dimensional structures. For the SSIM we calculated the metric band by band and took the average for a global characterisation. 


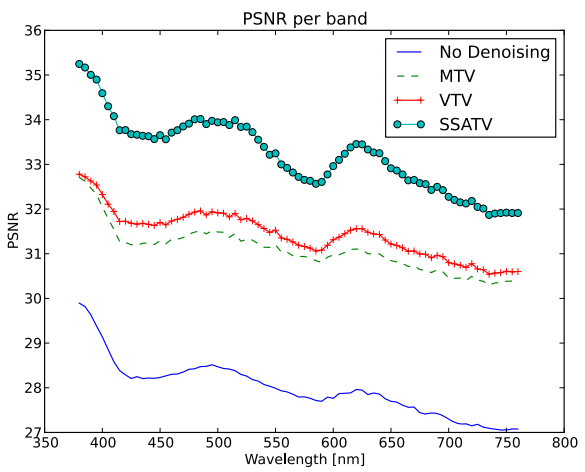

(a) PSNR band-wise

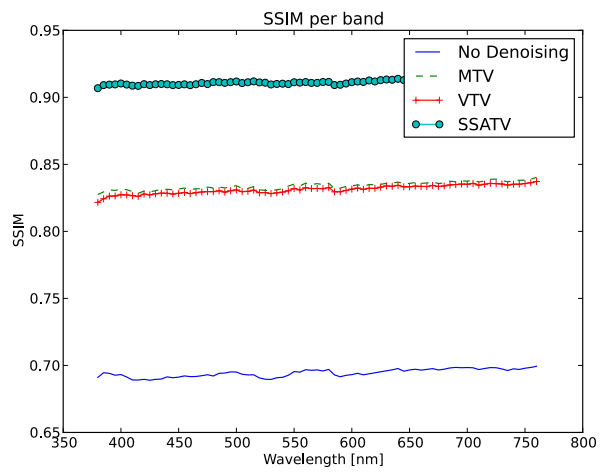

(b) SSIM band-wise

Fig. 2. Band-wise results PSNR, for noise level $\sigma_{4}=0.04$

Fig[2] shows band-wise results for both metrics for a medium noise level of $\sigma_{4}=$ 0.04. A better performance of SSATV is consistent over all bands. An improvement of VTV compared to MTV is only notable in terms of PSNR. All denoising approaches show a considerable improvement to a noise corrupted image.

Table 1 and Table 2 show the global results in terms of PSNR, respectively SSIM. In terms of PSNR, SSATV shows the best results for all noise levels. Compared to the noisy image, SSATV with $\sigma=0.08$ has roughly the same PSNR as the noisy image with noise intensity $\sigma=0.04$. The effects are less prominent on lower noise intensities. Regarding SSIM, MTV is beneficial in lower and the highest noise intensities.

Table 1. Results PSNR of different regularisation terms and noise levels

\begin{tabular}{lllllll}
\hline & \multicolumn{6}{c}{ Noise level } \\
\cline { 2 - 7 } & $\sigma_{1}=0.005$ & $\sigma_{2}=0.01$ & $\sigma_{3}=0.02$ & $\sigma_{4}=0.04$ & $\sigma_{5}=0.08$ & $\sigma_{6}=0.1$ \\
\hline Noisy & 46.0 & 40.0 & 34.0 & 28.0 & 22.0 & 20.0 \\
MTV & 47.7 & 42.2 & 36.4 & 31.0 & 26.7 & 25.7 \\
VTV & 47.0 & 41.6 & 36.4 & 31.4 & 27.0 & 25.8 \\
SSATV & $\mathbf{4 7 . 8}$ & $\mathbf{4 2 . 8}$ & $\mathbf{3 7 . 9}$ & $\mathbf{3 3 . 2}$ & $\mathbf{2 8 . 3}$ & $\mathbf{2 6 . 8}$ \\
\hline
\end{tabular}

Table 2. Results SSIM of different regularisation terms and noise levels

\begin{tabular}{lllllll}
\hline & \multicolumn{6}{c}{ Noise level } \\
\cline { 2 - 7 } & $\sigma_{1}=0.005$ & $\sigma_{2}=0.01$ & $\sigma_{3}=0.02$ & $\sigma_{4}=0.04$ & $\sigma_{5}=0.08$ & $\sigma_{6}=0.1$ \\
\hline Noisy & 0.990 & 0.962 & 0.876 & 0.694 & 0.469 & 0.405 \\
MTV & $\mathbf{0 . 9 9 7}$ & $\mathbf{0 . 9 8 9}$ & 0.947 & 0.834 & 0.728 & $\mathbf{0 . 6 9 7}$ \\
VTV & 0.993 & 0.978 & 0.936 & 0.834 & 0.700 & 0.640 \\
SSATV & 0.995 & 0.987 & $\mathbf{0 . 9 6 8}$ & $\mathbf{0 . 9 1 1}$ & $\mathbf{0 . 7 3 8}$ & 0.656 \\
\hline
\end{tabular}




\subsection{Real Dataset}

To evaluate the proposed denosing approach on a real dataset, we acquired a pigment test chart with a HySpex VNIR-1600 [1] hyperspectral scanner, that acquires 160 bands between $415 \mathrm{~nm}$ and $992 \mathrm{~nm}$, in a distance of $1 \mathrm{~m}$. To suppress signal-independent noise we averaged 10 measurement during the acquisition [1]. Fig 3 shows a small patch of $85 \mathrm{px} \times 100 \mathrm{px}$, that we used for a visual comparison. The top third shows the inhomogeneous granulate material, including some printed letters, the rest of the image shows two different pigments.

We compare the SSATV to the MTV approach, that have both shown the best result in the evaluation of the synthetic dataset. The algorithm is expected to reduce the noise especially in the pigment region, while preserving the prominent edge between the two regions. The parameters are adjusted that both approaches show a good result for $833 \mathrm{~nm}$.

It can be observed that MTV over-smoothes lower bands (433 nm) yet leaves a noisy result in higher bands $(960 \mathrm{~nm})$. SSATV model adapts for different noise intensities and preserves sharper edges, which is visible in the letters in top third.

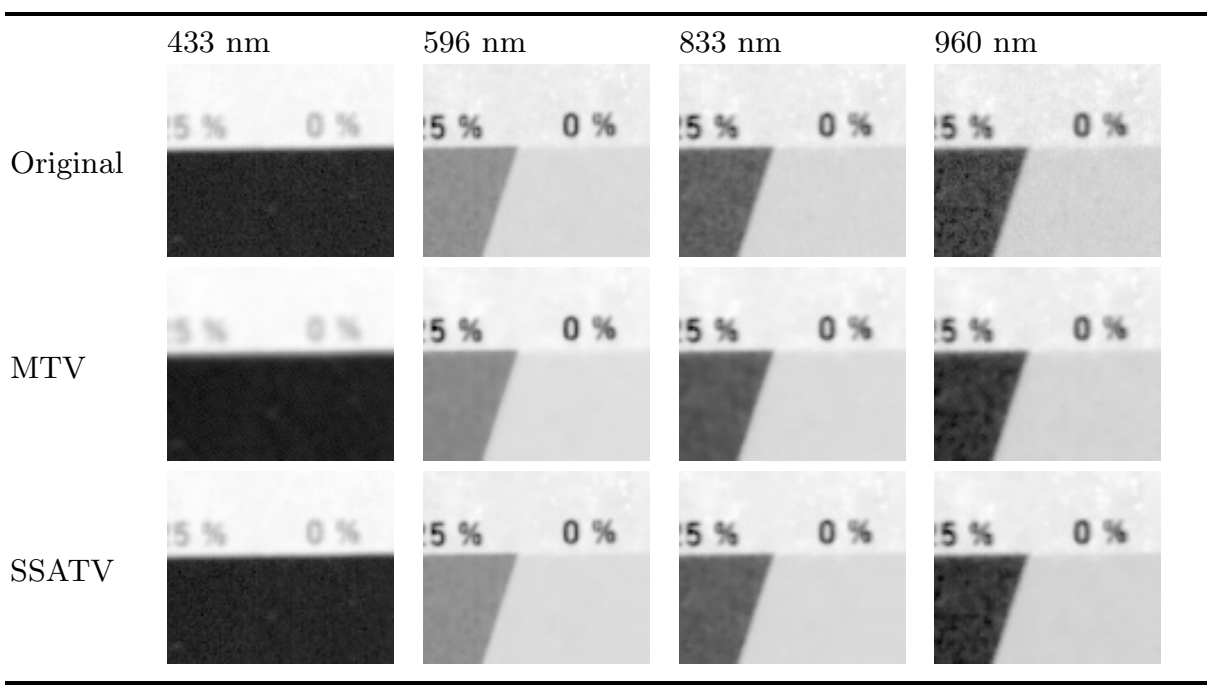

Fig. 3. The first line shows the acquisition of a HSI for four bands, the second and third line a denoised HSI for the same bands. SSATV adapts best to different bands, best visible in the first column.

\section{Conclusions and Further Work}

We proposed a variational approach to reconstruct HSI corrupted by poisson distributed noise. We showed that a ROF model with a spectral-spatial adaptive 
TV regularisation term performs better than a marginal approach regularisation. We evaluated our approach on a synthetic image with defined noise characteristics and on a real image with an unknown noise distribution.

Further research includes a comparison to other denoising approaches and an automatic parameter estimation. In a laboratory setup with a short distance acquisition, the parameters could be estimated by measuring a test target, with known properties.

Acknowledgement. This work is supported by the Regional Council of Burgundy. The hyperspectral scanner was gratefully provided by Norsk Elektro Optikk AS.

\section{References}

1. Imaging Spectrometer Users Manual, Norsk Elektro Optikk AS (2013)

2. Bregman, L.: The relaxation method of finding the common point of convex sets and its application to the solution of problems in convex programming. USSR Comput. Math. Math. Phys. 7(3), 200-217 (1967)

3. Bresson, X., Chan, T.: Fast dual minimization of the vectorial total variation norm and applications to color image processing. Inverse Probl. Imaging 2(4), 455-484 (2008)

4. Delcourt, J., Mansouri, A., Sliwa, T., Voisin, Y.: A Comparative Study and an Evaluation Framework of Multi/Hyperspectral Image Compression. In: Fifth Int. Conf. Signal Image Technol. Internet Based Syst., pp. 81-88 (November 2009)

5. Fairchild, M.D., Johnson, G.M.: METACOW: A Public-Domain, High- ExtendedDynamic-Range, Spectral Test Target for Imaging System Analysis and Simulation. In: Color Imaging Conf., pp. 239-245. IS\&T (2004)

6. Getreuer, P.: Rudin-Osher-Fatemi Total Variation Denoising using Split Bregman. Image Process. Line (3) (May 2012)

7. Krishnamurthy, K., Raginsky, M., Willett, R.: Multiscale Photon-Limited Spectral Image Reconstruction. SIAM J. Imaging Sci. 3(3), 619-645 (2010)

8. Le, T., Chartrand, R., Asaki, T.J.: A Variational Approach to Reconstructing Images Corrupted by Poisson Noise. J. Math. Imaging Vis. 27(3), 257-263 (2007)

9. Mansouri, A., Marzani, F., Gouton, P.: Development of a Protocol for CCD Calibration: Application to a Multispectral Imaging System. Int. J. Robot. Autom. 20(2), 81-88 (2005)

10. Mansouri, A., Sliwa, T., Hardeberg, J.Y., Voisin, Y.: An adaptive-PCA algorithm for reflectance estimation from color images. In: IEEE 19th Int. Conf. Pattern Recognit., vol. (1), pp. 1-4 (December 2008)

11. Osher, S., Goldstein, T.: The Split Bregman Method for L1-Regularized Problems. SIAM J. Imaging Sci. 2(2), 323-343 (2009)

12. Othman, H., Qian, S.E.: Noise Reduction of Hyperspectral Imagery Using Hybrid Spatial-Spectral Derivative-Domain Wavelet Shrinkage. IEEE Trans. Geosci. Remote Sens. 44(2), 397-408 (2006)

13. Rudin, L., Osher, S., Fatemi, E.: Nonlinear total variation based noise removal algorithms. Pysica D 60, 259-268 (1992) 


\section{4}

F. Deger et al.

14. Wang, Z., Bovik, A.C., Sheikh, H.R., Simoncelli, E.P.: Image quality assessment: from error visibility to structural similarity. IEEE Trans. Image Process. 13(4), 600-612 (2004)

15. Yuan, Q., Zhang, L., Member, S., Shen, H.: Hyperspectral Image Denoising Employing a Spectral Spatial Adaptive Total Variation Model. IEEE Trans. Geosci. Remote Sens. 50(10), 3660-3677 (2012)

16. Zanella, R., Boccacci, P., Zanni, L., Bertero, M.: Efficient gradient projection methods for edge-preserving removal of Poisson noise. Inverse Probl. 25(4) (April 2009) 